\title{
The Importance of Phenotyping Bronchiectasis
}

\author{
Salvatore Battaglia ${ }^{a} \quad$ Antoni Torres ${ }^{b}$ Eva Polverino ${ }^{b}$ \\ ${ }^{a}$ Sezione di Malattie Cardio-Respiratorie ed Endocrino-Metaboliche, Dipartimento Universitario Di.Bi.MIS, \\ University of Palermo, Palermo, Italy; ${ }^{b}$ Servei de Pneumologia, Hospital Clinic, Universitat de Barcelona, \\ Fundaciò Clìnic, IDIBAPS, CIBERES, Hospital Clinic de Barcelona, Barcelona, Spain
}

Bronchiectasis is considered to be one of the most heterogeneous respiratory diseases due to both multiple etiological conditions and variable clinical manifestations. This huge heterogeneity is one of the main reasons for disease complexity, and thus, there is a need to identify clinical phenotypes which have specific therapeutic and follow-up indications as in other respiratory diseases [i.e. chronic obstructive pulmonary disease (COPD) or asthma]. Unfortunately, identifying phenotypes of bronchiectasis is not an easy task, and so far, attempts to link aetiology to clinical severity have failed [1].

Aliberti et al. [2] performed an interesting cluster analysis that identified four phenotypes based on microbiological and clinical features ('Pseudomonas', 'other chronic infection', 'daily sputum' and 'dry bronchiectasis') but not on the different aetiologies of the disease. These phenotypes clearly showed different outcomes (quality of life, exacerbations, hospitalisations and mortality) and highlighted the importance of a more personalised approach to bronchiectasis.

More recently, Buscot et al. [3] presented a retrospective study aimed at describing the clinical, functional and microbiological phenotypes according to different aetiologies of bronchiectasis. This study has several strengths. First of all, the proposed diagnostic flowchart reduced the group classified as idiopathic to $11 \%$. The distinction between idiopathic bronchiectasis (i.e. unidentified aetiology after complete diagnostic workup) and 'undetermined' bronchiectasis (i.e. unidentified aetiology with incomplete diagnostic workup) seems more stringent and appropriate than in other publications from the past. Secondly, the proposed groups are related to the underlying aetiology of bronchiectasis, are easily identifiable and provide useful information for clinical management. Indeed, the authors identify nine aetiology groups: idiopathic, post-infectious, COPD-related, asthma-related, congenital, immunodeficiency, auto-immune, tumour and other. Among these, the COPD-related group clearly showed differential characteristics: an imbalance in gender ratio (male/female $=2.2$ ), the highest prevalence of smokers (as expected), the highest median age at diagnosis, a higher prevalence of Pseudomonas aeruginosa and the worst lung function at diagnosis. On the other hand, idiopathic bronchiectasis showed the highest prevalence of females (male/female $=0.23$ ) and the most preserved lung function at diagnosis. Finally, post-infectious bronchiectasis did not show distinguishable characteristics.

Numerous studies have investigated the combination of COPD and bronchiectasis, showing different prevalence rates (from 2 to $50 \%$ ) depending on cohort and primary diagnosis considered (COPD or bronchiectasis) [ 1 ,

\section{KARGER}

E-Mail karger@karger.com

www.karger.com/res
(C) 2016 S. Karger AG, Basel

0025-7931/16/0923-0134\$39.50/0
Dr. Eva Polverino

Servei de Pneumologia, Hospital Clinic, Universitat de Barcelona Fundaciò Clìnic, IDIBAPS, CIBERES, Hospital Clinic de Barcelona Calle Villarroel 170, ES-08036 Barcelona (Spain)

E-Mail epolveri@ clinic.ub.es 
$4,5]$. Although the impact of this overlap condition is not completely clear and possibly influenced by potential confounding factors (overall weight of comorbidities and related treatments), it seems clear that patients with COPD and bronchiectasis show more severe disease (lung function, chronic Pseudomonas, etc.) and a potentially increased risk of mortality [6].

The introduction of aetiologies into this classification may have the added value of considering some features that surely influence the clinical management of these patients. The reduced tolerance for inhaled antibiotics of COPD- and asthma-related bronchiectasis is for instance a quite specific feature of this group that may influence long-term prognosis. The other identified groups of immunodeficiencies, auto-immune disorders and tumour may also have differential therapeutic and prognostic implications that are surely worth considering in clinical practice.

One of the study limitations is that the retrospective nature of the study precludes the possibility to demonstrate a true 'cause-effect' in several groups; in this con- text, for instance, it is impossible to determine whether COPD or asthma truly preceded and prompted the development of bronchiectasis or, on the contrary, are associated with bronchiectasis as a functional expression of the airways inflammatory pattern. Moreover, the reported aetiology could be biased by the referral centre, and it cannot represent incident data from France or the rest of Europe. Nevertheless, we consider this study as a step forward on the way to a better understanding of the disease. Hopefully, a better knowledge of the prognostic factors and long-term outcomes across the different subsets or phenotypes of the disease may consistently change the future of our patients and change the current management toward a more personalised medicine approach. A good stratification of patients into different phenotypes, as in asthma or COPD, may help clinicians to individualise therapy and follow-up and hopefully positively influence the long-term prognosis of these patients. In addition, this is a crucial point when designing randomised clinical trials in order to include proper target populations and optimise their results.

\section{References}

$>1$ Lonni S, Chalmers JD, Goeminne PC, McDonnell MJ, Dimakou K, De SA, Polverino E, Van de KC, Rutherford R, Davison J, Rosales E, Pesci A, Restrepo MI, Torres A, Aliberti S: Etiology of non-cystic fibrosis bronchiectasis in adults and its correlation to disease severity. Ann Am Thorac Soc 2015;12:1764-1770.

$>2$ Aliberti S, Lonni S, Dore S, McDonnell MJ, Goeminne PC, Dimakou K, Fardon TC, Rutherford R, Pesci A, Restrepo MI, Sotgiu G, Chalmers JD: Clinical phenotypes in adult patients with bronchiectasis. Eur Respir J 2016; 47:1113-1122.
-3 Buscot M, Pottier H, Marquette C-H, Leroy S: Phenotyping adults with non-cystic fibrosis bronchiectasis: a 10-year cohort study in a French Regional University Hospital Center. Respiration 2016;92:1-8.

$\checkmark 4 \mathrm{Ni} \mathrm{Y,} \mathrm{Shi} \mathrm{G,} \mathrm{Yu} \mathrm{Y,} \mathrm{Hao} \mathrm{J,} \mathrm{Chen} \mathrm{T,} \mathrm{Song} \mathrm{H:}$ Clinical characteristics of patients with chronic obstructive pulmonary disease with comorbid bronchiectasis: a systemic review and meta-analysis. Int J Chron Obstruct Pulmon Dis 2015;10:1465-1475.
7 Divo M, Cote C, de Torres JP, Casanova C, Marin JM, Pinto-Plata V, Zulueta J, Cabrera C, Zagaceta J, Hunninghake G, Celli B: Comorbidities and risk of mortality in patients with chronic obstructive pulmonary disease. Am J Respir Crit Care Med 2012;186:155161.

6 Goeminne PC, Nawrot TS, Ruttens D, Seys S, Dupont LJ: Mortality in non-cystic fibrosis bronchiectasis: a prospective cohort analysis. Respir Med 2014;108:287-296. 